\title{
Effect of synchronization and semen sorting on artificial insemination bull fertility
}

\author{
G. Abdel-Azim ${ }^{1}$ \\ Genex Cooperative Inc., Shawano, WI 54166
}

\begin{abstract}
Field data were collected over a period of 2 yr by artificial insemination technicians for the purpose of evaluating differences among bulls in their fertility when synchronization and semen sorting were involved. First, main effects of synchronization and semen sorting were found to reduce bull fertility by 1.5 and $12.7 \%$, respectively. Second, the interaction of both factors with bull fertility significantly enhanced the evaluation models. Differences between 2 sets of adjusted conception rates for synchronized and nonsynchronized services ranged from 0.5 to $2.9 \%$, whereas differences between 2 sets of adjusted conception rates for sorted and conventional semen ranged from -1.8 to $15.2 \%$. This implies that using conventional fertility models that ignore these effects may not be sufficiently accurate in situations where synchronization or semen sorting are involved. Accounting for synchronization and especially for semen sorting to evaluate bulls on their fertility and the production of separate sets of conception rates under each situation are essential.
\end{abstract}

Key words: synchronization, sorted semen, bull fertility, nonreturn rate

\section{INTRODUCTION}

Bull fertility is difficult to evaluate in the laboratory because of the numerous stages that semen goes through before the actual insemination process occurs. General stages involve semen processing, freezing, storing, and thawing. These stages change many of the initial measures taken in the laboratory. In addition, most AI organizations have strict culling protocols based on various laboratory measures to cull inferior ejaculates and bulls, which leaves insufficient variation to analyze fertility based on data collected in the laboratory. In other cases these laboratory measures can be enhanced beyond major fertility limitations, as in the case of sperm cell concentration. Sperm cell concentration is

Received May 1, 2009.

Accepted September 30, 2009.

${ }^{1}$ Corresponding author: gamal.azim@gmail.com associated with fertility mostly when extremely small concentrations are considered (Foote and Kaproth, 1997; Den-Daas et al., 1998).

Because of the limitations of laboratory measures, actual breeding results, particularly nonreturn rate, have been used for decades to evaluate bulls for their fertility (e.g., Huston et al., 1953; Hopwood et al., 1956; Weigel and Rekaya, 2000; Clay and McDaniel, 2001). In the current study, when conception rate is mentioned in regard to a bull, it means the fertility of its semen as evaluated based on female nonreturn data.

Synchronization and semen sorting are 2 factors that negatively affect fertility. The literature is scarce for the effect of synchronization (Kuhn et al., 2008). However, the adverse effect of the current protocols of semen sorting is well documented (Seidel, et al., 1999; Weigel, 2004). The objective of this study was to evaluate the effect of synchronization and semen sorting on the fertility of processed semen of AI bulls. Extra attention was given to the interaction of bull fertility with synchronization and to the interaction of bull fertility with the process of semen sorting. The interactions may be important in identifying bulls that do not perform well under synchronization protocols and bulls that should not be used for semen sorting.

\section{MATERIALS AND METHODS}

\section{Field Data}

Data were collected over a period of 2 yr (March 2007-March 2009). Artificial insemination technicians employed by Genex/CRI (Shawano, WI) recorded the data during the AI process on farm. Factors recorded included date of insemination or breeding service; herd, bull, and cow identification numbers; AI technician; cow parity; and type of synchronization protocol, if any. In addition, a control number that traced semen to the processing laboratory was recorded.

Records used in the analysis were those from large herds (a minimum of 500 records/herd). In addition, herds were required to have at least $5 \%$ of their total collected services as synchronized services to be included in the analysis. Also, herds with average nonreturn rates 
$<10$ or $>90 \%$ were excluded from the analysis. Only Holstein bulls were considered in the current study.

Further edits included a minimum of 200 synchronized and 200 conventional services per bull, and a minimum of 1,000 total services per AI technician. Final data set included 543,491 services collected from 438 herds. This data set will be referred to as data set 1 .

The 2 synchronization protocols used in this study were Cosynch and Ovsynch and accounted for 25 and $75 \%$, respectively, of all synchronized services. Differences in unadjusted means between Cosynch and Ovsynch were essentially zero and the 2 categories were merged into 1 category of synchronized services. Synchronized services accounted for $27 \%$ of data set 1 .

To study the interaction effect of bull by semen sorting status, data set 2 was formed by retaining only herds with at least 500 total services but that used and confirmed at least 50 services of sorted semen. Only bulls that contributed at least 100 services of sorted semen were included in the analysis. Data set 2 was composed of 30,489 services of sorted semen and 158,275 services of conventional semen.

\section{Analysis}

To analyze bull fertility, observations were coded as 0 or 1 , where 1 indicated a successful conception after insemination. Success of a breeding was determined based on nonreturn rate (i.e., if a cow did not return for rebreeding for $59 \mathrm{~d}$, the cow was considered pregnant). If a cow returned for rebreeding after an interval that was less than $59 \mathrm{~d}$ and greater than $9 \mathrm{~d}$, the cow was considered open. Repeated services within $9 \mathrm{~d}$ were not considered as returns. Nonreturn data are different from actual conception data because a terminal service coded as 1 may also mean culling or death. However, the benefits gained by using all services overweigh the bias introduced by terminal services, particularly given that they constitute about $0.1 \%$ of nonreturn data (Abdel-Azim et al., 2004).

A generalized linear mixed model with logit link suitable for the dichotomous fertility data was fit. The model included synchronization status (synchronized and nonsynchronized), year-season, parity, semen sorting status (sorted and conventional), and service number as fixed factors. Random effects in the model included bull, bull by synchronization status interaction, herd, AI technician, and cow. The service number factor was included to adjust for large differences in cow fertility because cows that remained for third and following services would usually be less fertile than those that conceived at their first and second services.

To study the effect of bull by synchronization interaction, 2 models were compared by a likelihood ratio test, a full model as described above (full model I), and a reduced model without the bull by synchronization interaction.

A similar approach was followed to study the interaction effect of bull by semen sorting. The interaction was added as a random effect to the reduced model described above and a second likelihood ratio test to compare the 2 models was used. The second full model with the interaction effect of bull by semen sorting will be referred to as full model II.

\section{Probability of Conception Rates}

To transform solutions to the observable probability scale, the inverse link function, $\exp (x) /[1+\exp (x)]$, was evaluated, where $x$ is a linear combination of effects on the linear scale. To estimate the difference between any 2 levels of a factor, effects of the 2 levels were transformed first to the probability scale and then the difference was calculated (McCullagh and Nelder, 1989; Littell et al., 1999).

Conception rate (CR) of a bull was calculated after an appropriate set of probabilities were estimated. For example, to calculate a bull's CR value for synchronized services, all linear combinations across the model fixed effects were calculated. The linear effect of the bull, its interaction effect with synchronized services, and the solution of the fixed effect associated with synchronized services were added to each combination. A transformation to the probability scale was then performed by the inverse link function described above. Harville and Mee (1984) and Gianola and Foulley (1983) discussed in detail transforming linear estimates to the observable scale.

\section{RESULTS}

\section{Data Summaries}

Tables 1 and 2 list unadjusted nonreturn means associated with fixed factors used in the analysis of data sets 1 and 2, respectively. Table 1 shows an overall drop in fertility of about $2 \%$ with synchronized services. The unadjusted difference between synchronized and nonsynchronized services was more pronounced in first and second services than in subsequent services. The table shows better unadjusted means for synchronized services within heifers and cows, which may seem contradictory to the lower unadjusted mean of synchronized services in general. However, by considering weighted means of heifer and cow services within the 2 synchronization status categories, we obtained the tabulated general means for the 2 categories. Nonsynchronized heifer and cow services were 73,340 and 325,753 , respectively, and 
Table 1. Unadjusted means for nonreturn rates across fixed factors used in the analysis of data set 1

\begin{tabular}{lcc}
\hline Item & $\begin{array}{c}\text { Nonsynchronized } \\
\text { services }\end{array}$ & $\begin{array}{c}\text { Synchronized } \\
\text { services }\end{array}$ \\
\hline All data & 50.50 & 48.58 \\
Parity & 62.67 & 65.80 \\
$\quad$ Heifers & 47.77 & 48.22 \\
$\quad$ Cows & 52.90 & 50.00 \\
Semen sorting status & 50.43 & 48.57 \\
$\quad$ Sorted & & \\
$\quad$ Conventional & 57.66 & 53.30 \\
Service number & 52.14 & 50.12 \\
$\quad$ 2 & 44.33 & 44.12 \\
$\quad \geq 3$ & 47.73 & 45.06 \\
Year-season range & 54.22 & 53.45 \\
$\quad$ From & & \\
To & & \\
\hline
\end{tabular}

synchronized heifer and cow services were 2,939 and 141,459 , respectively.

Tables 1 and 2 show higher nonreturn unadjusted means for sorted than conventional semen. The reason is because sorted semen was used more on heifers than on cows; $85 \%$ of the sorted semen was used on heifers. The statistical analysis, however, adjusted for this because a 2-level parity factor to differentiate between heifer and cow services accompanied the sorted semen factor in the model of analysis. DeJarnette et al. (2009) reported that simple on-farm comparisons of sorted and conventional semen could be misleading because of using sorted semen more on the younger, more fertile females. Also, about $19 \%$ of all conventional semen was used on heifers where all heifer services accounted for $29 \%$ of the total number of services in data set 2 (Table $3)$.

Table 2. Unadjusted means for nonreturn rates across fixed factors used in the analysis of data set 2

\begin{tabular}{lcc}
\hline Item & $\begin{array}{c}\text { Conventional } \\
\text { semen services }\end{array}$ & $\begin{array}{c}\text { Sex-sorted semen } \\
\text { services }\end{array}$ \\
\hline All data & 50.36 & 52.06 \\
Parity & 63.95 & 53.44 \\
$\quad$ Heifers & 47.23 & 45.98 \\
$\quad$ Cows & 47.07 & 55.32 \\
Synchronization status & 50.98 & 51.94 \\
$\quad$ Synchronized & & \\
$\quad$ Nonsynchronized & 57.91 & 56.99 \\
Service number & 52.94 & 50.78 \\
1 & 43.84 & 40.08 \\
2 & & 49.20 \\
$\quad \geq 3$ & 48.09 & 55.90 \\
Year-season range & 55.00 & \\
From & & \\
To & &
\end{tabular}

Table 3. Number of confirmed nonreturn services in data sets 1 and 2 shown for classes of synchronization and sexed semen statuses, and for heifers and cows

\begin{tabular}{lcc}
\hline Item & Data set 1 & Data set 2 \\
\hline Synchronized & 144,398 & 26,171 \\
Nonsynchronized & 399,093 & 162,593 \\
Sex-sorted & 12,329 & 30,489 \\
Conventional & 531,162 & 158,275 \\
Heifers & 76,279 & 54,502 \\
Cows & 467,212 & 134,262 \\
\hline
\end{tabular}

\section{Full Model I}

Table 4 lists fixed effect solutions except for yearseason. Results in the table show a negative effect of synchronized versus nonsynchronized services on fertility. The effect on the linear scale was -0.0634 , which translates to a drop in fertility of $1.53 \%$ on the probability scale. The effect of synchronization was small when compared with the effect of other factors included in the model (Table 4). Average effect of other factors was as follows: adjusted conception rate for heifers was greater than that of cows by $15 \%$, and adjusted conception rate for conventional semen was greater than that of sorted semen by $12.7 \%$, unlike the trend in unadjusted means reported in Table 1. Finally, adjusted conception rates for service numbers 1,2 , and $\geq 3$ were $59.25,54.7$, and 50.07, respectively.

Variance components of random effects are listed in Table 5 along with their standard errors. Herd contributed the most to the variability in fertility, which emphasizes the importance of management practices to fertility. Fertility varied more with AI technicians than with bulls, which indicates that training and skill to apply AI were more important than the bulls involved.

\section{Reduced Model}

To study the significance of bull by synchronization interaction, a reduced model without interaction was fit. The 2 models were then compared and the full model was found to significantly reduce the residual

Table 4. Estimates of conception rates and SE for fixed effects, ${ }^{1}$ full model I

\begin{tabular}{lccc}
\hline Fixed factor & Estimate & $\mathrm{SE}$ & $\operatorname{Pr}(>|\mathrm{z}|)$ \\
\hline Overall mean $_{\text {Synchronization }}{ }^{2}$ & -0.14418 & 0.03506 & $<0.001$ \\
Heifers & -0.0634 & 0.00890 & $<0.001$ \\
Conventional semen & 0.62617 & 0.00983 & $<0.001$ \\
Service no. & 0.52883 & 0.02149 & $<0.001$ \\
\hline${ }^{1}$ Year-season solutions averaged 0.158 and ranged from & -0.002 to \\
0.427. & &
\end{tabular}


Table 5. Variance components of the random effects of full model I when applied to nonreturn data set 1

\begin{tabular}{lcc}
\hline Random factor & Variance component & SE \\
\hline Herd & 0.22177 & 0.01570 \\
Bull by synchronization interaction & 0.00079 & 0.00042 \\
AI technician & 0.01374 & 0.00211 \\
Bull & 0.00708 & 0.00136 \\
Cow & 0.17603 & 0.00452 \\
\hline
\end{tabular}

deviance $\left[\operatorname{Pr}\left(>\chi^{2}\right)=0.017\right]$. The significant interaction indicates that bulls may show different trends in fertility based on whether or not the inseminated cow was synchronized. This was further tested by comparing adjusted conception rates for bulls between synchronized and nonsynchronized services.

\section{Comparing Synchronized and Nonsynchronized Conception Rates}

Two sets of adjusted conception rates were obtained, one for synchronized and the other for nonsynchronized services. For each bull, the difference between his 2 conception rates for synchronization and nonsynchronization statuses was calculated. The 2 sets differed by a range of 0.5 to $2.9 \%$ across bulls. In addition, bulls were ranked based on the 2 sets of conception rates; differences in ranking ranged from -24 to 36 among 117 bulls included in the study. Table 6 summarizes details of change in rank based on synchronized and nonsynchronized services. The table shows that $19 \%$ of the bulls changed rank by at least 10 places up or down.

\section{Interaction Effect of Bull by Semen Sorting}

When the interaction effect of bull by semen sorting was added as a random effect to the reduced model described above and the 2 models were compared for the same data set (data set 2), the full model was found to significantly reduce residual deviance $\left[\operatorname{Pr}\left(>\chi^{2}\right)<\right.$ 0.0001]. The reduction in deviance was much greater than what was obtained earlier by fitting full model

Table 6. Differences in bull rankings across 2 sets of probability of conception rates estimated by transforming 2 linear functions to the observed probability scale of bulls when used with synchronized and nonsynchronized services

\begin{tabular}{lc}
\hline No. of bulls ${ }^{1}$ & Absolute change in rank \\
\hline 1 & $>30$ \\
4 & $>20$ \\
22 & $>10$ \\
51 & $>5$ \\
\hline
\end{tabular}

${ }^{1}$ Out of 117 bulls included in the analysis.
I. Variance components for full model II are listed in Table 7. It is apparent from Table 7 that the interaction variance in full model II was as important as the bull variance, which suggests that conventional bull fertility evaluations (e.g., Kuhn and Hutchison, 2008) incorrectly rank bulls for their sorted semen fertility. Discussion of discrepancies in bull rankings between conventional and sorted semen follows.

\section{Comparing Sorted and Conventional Conception Rates}

Two sets of adjusted conception rates were produced for sorted and conventional semen. The magnitude of the difference between the 2 sets was expected to be large because of the large difference between sorted and conventional semen effects. The difference of adjusted $\mathrm{CR}$ for conventional semen minus adjusted $\mathrm{CR}$ for sorted semen averaged $7.0 \%$ and ranged from -1.8 to $15.2 \%$.

The large difference between the 2 sets of CR does not indicate by itself that they do not predict each other very well. This is because the difference could be similar across bulls. This, however, was not the case because the correlation between them was only 0.66 . In addition, the correlation between the estimated interaction effects of sex-sorted and conventional semen was -0.47 . An interaction plot of 2 sets of linear effects of bulls for sex-sorted and conventional semen is given in Figure 1. A linear effect in the first set is the sum of a bull's predicted effect and the corresponding predicted interaction effect for sex-sorted semen, and a linear effect in the other set is the sum of a bull's predicted effect and the corresponding predicted interaction effect for conventional semen in full model II.

\section{DISCUSSION}

The negative effect of each of hormonal synchronization and the process of semen sorting should not be misunderstood as warnings against synchronization or semen sorting. This is why interaction components were fit in the models of analysis. The significant interaction between the process of semen sorting and bulls

Table 7. Variance components of the random effects of full model II when applied to nonreturn data set 2

\begin{tabular}{lcc}
\hline Random factor & Variance component & SE \\
\hline Herd & 0.27693 & 0.02843 \\
Bull by semen sorting interaction & 0.01489 & 0.00566 \\
AI technician & 0.03362 & 0.00556 \\
Bull & 0.01530 & 0.00702 \\
Cow & 0.08694 & 0.00930 \\
\hline
\end{tabular}




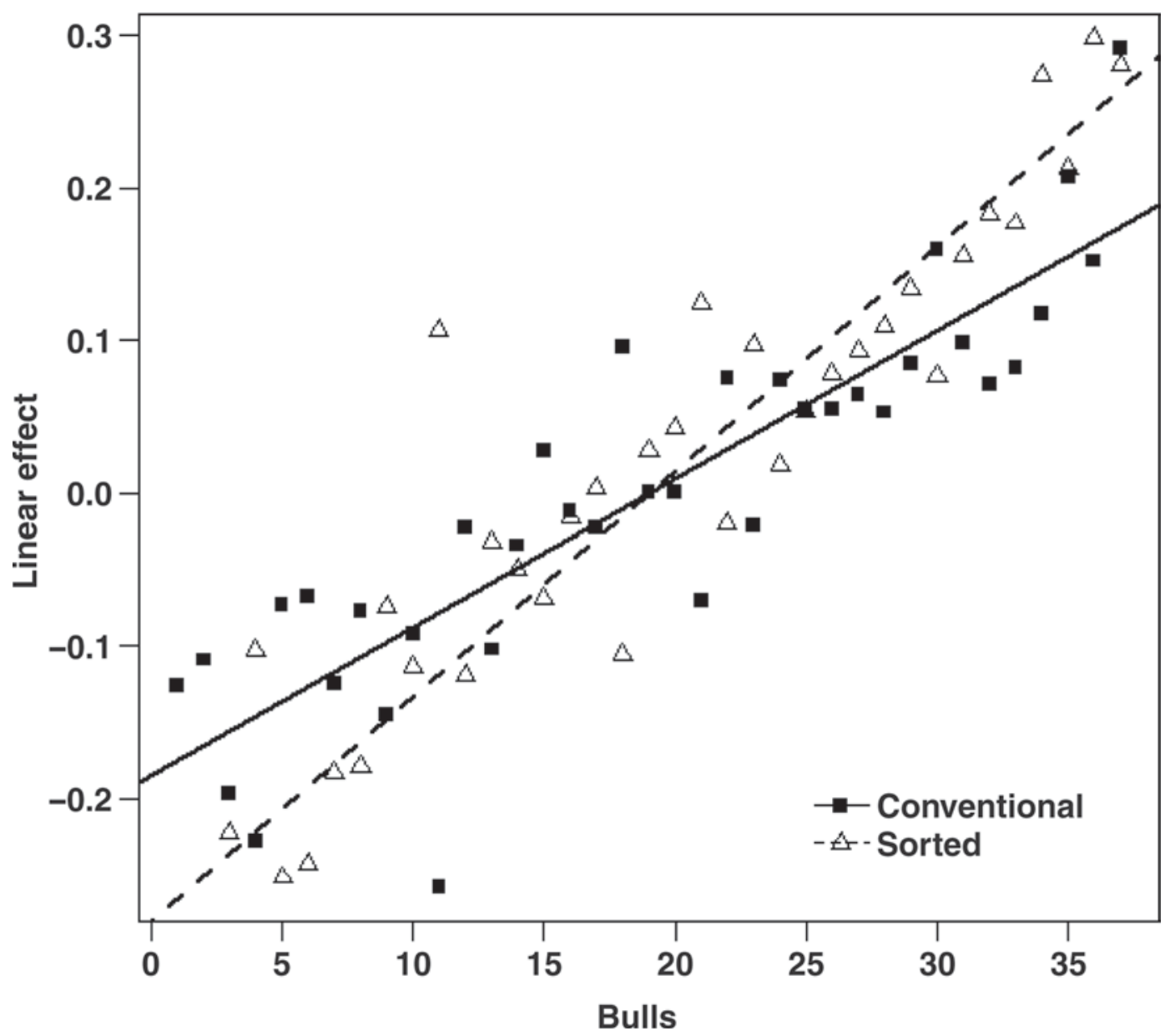

Figure 1. Interaction plot of 2 sets of bull linear effects for sex-sorted and conventional semen where the 2 predicted interaction effects of a bull with sex-sorted and conventional semen were added to the predicted main effect of the bull.

means that bulls were not the same in their reaction to the process. Hence, bulls have to be selected based on a specialized analysis before subjecting their sperm cells to the process of semen sorting. The same can be said about synchronization protocols, either to rank bulls based on an independent analysis of synchronized services or to add synchronization and its interaction with bull as extra components to the current models of analysis that operate with mixed data of both synchronized and nonsynchronized services. This study, however, followed the second approach, which was to add synchronization and its interaction to the basic model, and that was also the case with sorted semen.

The single-model approach followed in this study might be appropriate in situations where a single reliability value per bull is needed for its 2 types of evaluations. As described in this study, the steps involved to produce 2 types of evaluations, one appropriate for ranking bulls for synchronized and the other for nonsynchronized cows, can be summarized as follows: 1) run the full model that includes synchronization status and its interaction with bull; 2) produce solutions for model effects; 3 ) use appropriate combinations to calculate the 2 types of bull estimates (the first type is calculated by adding to bull solutions estimates of the synchronization category and bull by synchronization interaction; the second type includes bull solutions as before, but in this case, estimates of the nonsynchronization category and bull by nonsynchronization interaction are added); and 4) transform both sets to the probability scale by the inverse linking appropriate to the model used to produce solutions. The singlemodel approach guarantees equal reliabilities for bulls because the same bull solutions are used in the 2 types of bull fertility evaluations. Step 4 can be as sophisticated as one wishes; for example, if standard errors of the model effects produced in step 2 and used in step 3 were small for some levels and large for others, then a specific weighing based on these standard errors can be integrated into step 4 (Gianola and Foulley, 1983; 
Harville and Mee, 1984; Abdel-Azim et al. 2004). The same approach can be followed for bull evaluations for sorted and conventional semen.

Further research is still needed to explain why hormonal synchronization reduces fertility and why sperm cells react differently to the adverse effect of semen sorting. This study, based on wealth of data collected over a period of $2 \mathrm{yr}$, should constitute a good foundation for further research in reproductive physiology.

\section{CONCLUSIONS}

This study assessed the effect of synchronization and semen sorting on fertility using nonreturn data collected over a period of $2 \mathrm{yr}$. Although the average effect of synchronization and semen sorting is known to negatively affect fertility, the current analysis showed significant interactions between these 2 important factors and bull fertility. This indicates that bulls react differently to synchronization and semen sorting. The interaction effect of semen sorting was large and contributed to variability in fertility as much as the contribution of bulls. This indicates that current bull fertility evaluations based only on conventional semen may incorrectly rank bulls for their sorted semen fertility. Separate evaluations for bulls for their sorted semen and synchronized services are needed, or, if data permit, proper modifications to current fertility models may be pursued.

\section{REFERENCES}

Abdel-Azim, G., S. Schnell, G. Gilbert, and H. Rycroft. 2004. Genetic evaluation of male fertility using a threshold model with emphasis on accurate estimation of conception rate. J. Dairy Sci. 87(Suppl. 1):242. (Abstr.)

Clay, J. S., and B. T. McDaniel. 2001. Computing mating bull fertility from DHI nonreturn data. J. Dairy Sci. 84:1238-1245.

DeJarnette, J. M., R. L. Nebel, and C. E. Marshall. 2009. Evaluating the success of sex-sorted semen in US dairy herds from on farm records. Theriogenology 71:49-58.

Den-Daas, J. H. G., G. De-Jong, L. M. T. E. Lansbergen, and A. M. Van Wagtendonk-De Leeuw. 1998. The relationship between the number of spermatozoa inseminated and the reproductive efficiency of individual dairy bulls. J. Dairy Sci. 81:1714-1723.

Foote, R. H., and M. T. Kaproth. 1997. Sperm numbers inseminated in dairy cattle and nonreturn rates. J. Dairy Sci. 80:3072-3076.

Gianola, D., and J. L. Foulley. 1983. Sire evaluation for ordered categorical data with a threshold model. Genet. Sel. Evol. $15: 201-224$.

Harville, D. A., and R. W. Mee. 1984. A mixed-model procedure for analyzing ordered categorical data. Biometrics 40:393-408.

Hopwood, M. L., E. R. Rutherford, and F. X. Gassner. 1956. The concept of fructose utilization by bull sperm and its relation to fertility. J. Dairy Sci. 39:51-59.

Huston, K. A., K. J. Arnold, E. E. Heizer, and N. D. Bayley. 1953. A systematic procedure for estimating bull fertility from non-return data. J. Dairy Sci. 36:260-267.

Kuhn, M. T., and J. L. Hutchison. 2008. Prediction of dairy bull fertility from field data: Use of multiple services and identification and utilization of factors affecting bull fertility. J. Dairy Sci. 91:2481-2492.

Kuhn, M. T., J. L. Hutchison, and H. D. Norman. 2008. Modeling nuisance variables for prediction of service sire fertility. J. Dairy Sci. 91:2823-2835.

Littell, R. C., G. A. Milliken, W. W. Stroup, and R. D. Wolfinger. 1999. SAS System for Mixed Models. SAS Institute Inc., Cary $\mathrm{NC}$

McCullagh, P., and J. A. Nelder. 1989. Generalized Linear Models. 2nd ed. Chapman \& Hall, NewYork, NY.

Seidel, G. E. Jr., J. L. Schenk, L. A. Herickhoff, J. L. Schenk, S. P. Doyle, and Z. Brink. 1999. Insemination of heifers with sexed semen. Theriogenology 52:1407-1420.

Weigel, K. A. 2004. Exploring the role of sexed semen in dairy production systems. J. Dairy Sci. 87(E-Suppl.):E120-E130.

Weigel, K. A., and R. Rekaya. 2000. Genetic parameters for reproductive traits of Holstein cattle in California and Minnesota. J. Dairy Sci. 83:1072-1080. 